\title{
Biodiesel: Métodos de Análise e sua Influência em Materiais Metálicos e Poliméricos
}

\section{Luciano M. Lião, Marcos R. Monteiro e Alessandra R. P. Ambrozin}

Diversos fatores têm impulsionado o uso de combustíveis alternativos em substituição aos derivados de petróleo. O biodiesel é a principal alternativa ao diesel fóssil. $\mathrm{O}$ sucesso da sua utilização como combustível está intimamente ligado ao seu padrão de qualidade, que se relaciona não somente ao controle da sua composição química como também à influência desse biocombustível sobre os materiais que constituem os sistemas veiculares, de transporte e armazenamento. Nesse trabalho, são descritos os principais métodos analíticos utilizados no controle de qualidade do biodiesel e de suas misturas com diesel, assim como o efeito do biodiesel sobre materiais metálicos e poliméricos.

Palavras-chave: biodiesel; métodos analíticos; metais; elastômeros.

The widespread use of alternative fuels to derived-petroleum ones has increased due to several factors. Biodiesel is the major substitute to fossil diesel. Its successful utilization as a fuel is close related to its quality, which is associated to control of its chemical composition as well as its effect on materials that constitute engines and transportation and storage systems. In this work, the main analytical methods used for the quality control of biodiesel and its mixtures with diesel, as well as the effect of biodiesel on metals and polymer materials are described.

Keywords: biodiesel; analytical methods; metals; elastomers. 


\section{Introdução}

O uso de biodiesel como combustível alternativo ao diesel fóssil tem aumentando nos últimos anos em virtude de questões de preservação ambiental e conservação de energia, assim como para o fortalecimento da base agroindustrial e o incremento da sustentabilidade da matriz energética. Originário de fonte renovável, sua utilização em misturas com diesel implica na redução das principais emissões associadas aos derivados de petróleo. ${ }^{1}$ As emissões de gases associados ao efeito estufa do biodiesel puro (B100) metílico de soja e colza foram avaliadas na última década e os resultados indicaram reduções de 40 a $60 \%$ nessas emissões, com exceção dos óxidos de nitrogênio (NOx). ${ }^{2}$

O óleo diesel pode ser complementado com biodiesel para uso nos motores a diesel e nos sistemas de transporte e armazenamento sem que sejam necessárias alterações ${ }^{1,3}$. Entretanto, os altos custos de produção, a baixa estabilidade oxidativa, o aumento da emissão dos NOx e a aplicação dos grandes volumes de co-produtos (glicerina e biomassa) são aspectos que ainda devem ser avaliados. $^{4-7}$

O biodiesel, constituído majoritariamente por ésteres alquílicos de ácidos graxos, é produzido a partir de diversas matérias-primas, tais como óleos vegetais, gorduras animais, óleos e gorduras residuais. Ele é produzido através de diversos processos, especialmente através de transesterificação, que consiste na reação dos triglicerídeos, presentes em óleos e gorduras, com um álcool (metanol, etanol, etc) na presença de um catalisador ácido ou básico. Freqüentemente, a transesterificação é realizada com metanol sob catálise homogênea básica $\left(\mathrm{NaOH}, \mathrm{KOH}, \mathrm{NaOCH}^{3}, \mathrm{KOCH}^{3}\right.$ ) (Figura 1). ${ }^{8-10} \mathrm{O}$ glicerol (ou glicerina) é o principal co-produto dessa reação, mas também mono-, di-, triglicerídeos, ácidos graxos livres, água, álcool, catalisador residual e outras impurezas podem constituir o produto final. ${ }^{9} \mathrm{O}$ conteúdo de tais impurezas está diretamente relacionado com a qualidade do biodiesel. Os parâmetros de qualidade são estabelecidos em normas de padronização, visando assegurar a alta qualidade do produto final e o uso seguro do biodiesel como combustível de motores de combustão interna.

$\mathrm{Na}$ Europa, as especificações foram estabelecidas na norma EN 14214 e nos EUA, na ASTM D 6751.11,12
No Brasil, as especificações do biodiesel (B100) foram definidas pela Resolução $n^{\circ} 42$ (de 24/11/2004) da ANP (Agência Nacional do Petróleo, Gás Natural e Biocombustíveis) e do B2 pela resolução $\mathrm{n}^{0} 15$ (de 17/07/2006). A designação B100 é usada para o biodiesel puro e, para misturas, utiliza-se a denominação BXX, que indica a porcentagem $\mathrm{XX}$, em volume, de biodiesel na mistura. Por exemplo, B2 possui 2\% (v/v) de biodiesel e $98 \%$ (v/v) de diesel; B5, $5 \%$ (v/v) de biodiesel e 95\% (v/v) de diesel. ${ }^{1,9}$ No Brasil, segundo a legislação em vigor (lei 11.097/2005 de 13/01/2005), a utilização de B2 será obrigatória a partir de 2008 e de B5, em 2013.

Para atender à norma brasileira, o biodiesel (B100) deve satisfazer diversas especificações descritas na Tabela 1. Nas normas de padronização também estão estabelecidas as metodologias de ensaio para a determinação dos parâmetros de qualidade. No Brasil, a determinação das características do biodiesel deve atender aos requisitos estabelecidos principalmente pelo emprego das normas da Associação Brasileira de Normas Técnicas (ABNT), da American Society for Testing and Materials (ASTM), da International Organization for Standardization (ISO) e do Comité Européen de Normalisation (CEN).

A obtenção de biodiesel que obedeça às regulamentações técnicas depende significativamente da qualidade da matéria-prima, do catalisador e das condições empregadas na reação de transesterificação, assim como do processo de purificação. Além disso, devido à baixa estabilidade do biodiesel, que pode sofrer reações de hidrólise e oxidação, condições adequadas de transporte e armazenamento são essenciais para a manutenção das especificações do biodiesel. ${ }^{13}$ Desta forma, a qualidade do biodiesel, que é inferida a partir de sua composição química e de alguns parâmetros físico-químicos, pode ser influenciada por fatores tais como composição da matériaprima, processo produtivo, transporte e armazenamento. Os parâmetros mais críticos no controle de qualidade do biodiesel referem-se à: conversão completa para os ésteres alquílicos; remoção da glicerina, catalisador e álcool; e ausência dos ácidos graxos. ${ }^{14}$

A determinação do conteúdo de biodiesel em suas misturas com diesel é também um aspecto relevante já que a comercialização dessas misturas tem aumentado significativamente nos últimos anos. ${ }^{11}$ 


\section{Artigo 3}

Tabela 1: Especificações do B100 estabelecidas pela resolução ${ }^{\circ} 42$ (de 24/11/2004) da ANP.

\begin{tabular}{|c|c|c|c|c|c|}
\hline \multirow{2}{*}{ CARACTERÍSTICA } & \multirow{2}{*}{ UNIDADE } & \multirow{2}{*}{ LIMITE } & \multicolumn{3}{|c|}{ MÉTODO } \\
\hline & & & ABNT NBR & ASTM D & EN/ISO \\
\hline Aspecto & - & LII (1) & - & - & - \\
\hline Massa específica a $20^{\circ} \mathrm{C}$ & $\mathrm{kg} / \mathrm{m} 3$ & Anotar (2) & 7148,14065 & 1298,4052 & - \\
\hline Viscosidade Cinemática a $40^{\circ} \mathrm{C}$ & $\mathrm{Mm} 2 / \mathrm{s}$ & Anotar (3) & 10441 & 445 & EN ISO 3104 \\
\hline Água e sedimentos, máx. (4) & $\%$ volume & 0,050 & - & 2709 & - \\
\hline Contaminação Total (6) & $\mathrm{mg} / \mathrm{kg}$ & Anotar & - & - & EN 12662 \\
\hline \multirow{2}{*}{ Ponto de fulgor, mín. } & \multirow{2}{*}{${ }^{\circ} \mathrm{C}$} & \multirow{2}{*}{100,0} & 14598 & 93 & - \\
\hline & & & - & - & EN ISO3679 \\
\hline Teor de éster (6) & $\%$ massa & Anotar & - & - & EN 14103 \\
\hline Destilação; 90\% vol. recuperados, máx. & ${ }^{\circ} \mathrm{C}$ & $360(5)$ & - & 1160 & - \\
\hline \multirow{2}{*}{ Resíduo de carbono dos $100 \%$ destilados, máx. } & \multirow{2}{*}{$\%$ massa } & \multirow{2}{*}{0,10} & - & 4530 & EN ISO 10370 \\
\hline & & & - & 189 & - \\
\hline Cinzas sulfatadas, máx. & $\%$ massa & 0,020 & 9842 & 874 & ISO 3987 \\
\hline \multirow{2}{*}{ Enxofre total (6) } & \multirow{2}{*}{$\%$ massa } & \multirow{2}{*}{ Anotar } & - & 4294 & - \\
\hline & & & - & 5453 & EN ISO 14596 \\
\hline \multirow{2}{*}{ Sódio + Potássio, máx } & \multirow{2}{*}{$\mathrm{mg} / \mathrm{kg}$} & \multirow{2}{*}{10} & - & - & EN 14108 \\
\hline & & & - & - & EN 14109 \\
\hline Cálcio + Magnésio (6) & $\mathrm{mg} / \mathrm{kg}$ & Anotar & - & - & EN 14538 \\
\hline Fósforo (6) & $\mathrm{mg} / \mathrm{kg}$ & Anotar & - & 4951 & EN 14107 \\
\hline Corrosividade ao cobre, $3 \mathrm{~h}$ a $50^{\circ} \mathrm{C}$, máx. & - & 1 & 14359 & 130 & EN ISO 2160 \\
\hline Número de Cetano (6) & - & Anotar & - & 613 & EN ISO 5165 \\
\hline Ponto de entupimento de filtro a frio, máx. & ${ }^{\circ} \mathrm{C}$ & (7) & 14747 & 6371 & - \\
\hline \multirow{2}{*}{ Índice de acidez, máx. } & \multirow{2}{*}{$\mathrm{mg} \mathrm{KOH} / \mathrm{g}$} & \multirow{2}{*}{0,80} & 14448 & 664 & - \\
\hline & & & - & - & EN $14104(8)$ \\
\hline \multirow{3}{*}{ Glicerina livre, máx. } & \multirow{3}{*}{$\%$ massa } & \multirow{3}{*}{0,02} & - & $6584(8)(9)$ & - \\
\hline & & & - & - & EN $14105(8)(9)$ \\
\hline & & & - & - & EN $14106(8)(9)$ \\
\hline \multirow{2}{*}{ Glicerina total, máx. } & \multirow{2}{*}{$\%$ massa } & \multirow{2}{*}{0,38} & - & $6584(8)(9)$ & - \\
\hline & & & - & - & EN 14105 (8) (9) \\
\hline \multirow{2}{*}{ Monoglicerídeos (6). } & \multirow{2}{*}{$\%$ massa } & \multirow{2}{*}{ Anotar } & - & $6584(8)(9)$ & - \\
\hline & & & - & - & EN 14105 (8) (9) \\
\hline
\end{tabular}


Tabela 1: Especificações do B100 estabelecidas pela resolução no 42 (de 24/11/2004) da ANP - Continuação.

\begin{tabular}{|c|c|c|c|c|c|}
\hline \multirow{2}{*}{ CARACTERÍSTICA } & \multirow{2}{*}{ UNIDADE } & \multirow{2}{*}{ LIMITE } & \multicolumn{3}{|c|}{ MÉTODO } \\
\hline & & & ABNT NBR & ASTM D & EN/ISO \\
\hline \multirow{2}{*}{ Diglicerídeos (6) } & \multirow{2}{*}{$\%$ massa } & \multirow{2}{*}{ Anotar } & - & $6584(8)(9)$ & - \\
\hline & & & - & - & EN 14105 (8) (9) \\
\hline \multirow{2}{*}{ Triglicerídeos (6) } & \multirow{2}{*}{$\%$ massa } & \multirow{2}{*}{ Anotar } & - & $6584(8)(9)$ & - \\
\hline & & & - & - & EN 14105 (8) (9) \\
\hline Metanol ou Etanol, máx. & $\%$ massa & 0,5 & - & - & EN $14110(8)$ \\
\hline Índice de Iodo (6) & & Anotar & - & - & EN $14111(8)$ \\
\hline Estabilidade à oxidação a $110^{\circ} \mathrm{C}$, min. & $\mathrm{h}$ & 6 & - & - & EN $14112(8)$ \\
\hline
\end{tabular}

NOTA:

1. LII - Límpido e isento de impurezas.

2. A mistura óleo diesel/biodiesel utilizada deverá obedecer aos limites estabelecidos para massa específica a $20^{\circ} \mathrm{C}$ (constantes da especificação vigente da ANP de óleo diesel automotivo).

3. A mistura óleo diesel/biodiesel utilizada deverá obedecer aos limites estabelecidos para viscosidade a $40^{\circ} \mathrm{C}$ (constantes da especificação vigente da $\mathrm{ANP}$ de óleo diesel automotivo).

4. O método EN ISO12937 poderá ser utilizado para quantificar a água não dispensando a análise e registro do valor obtido para água e sedimentos pelo método ASTM D 2709 no Certificado da Qualidade.

5. Temperatura equivalente na pressão atmosférica.

6. Estas características devem ser analisadas em conjunto com as demais constantes da tabela de especificação a cada trimestre civil. Os resultados devem ser enviados pelo produtor de biodiesel à ANP, tomando uma amostra do biodiesel comercializado no trimestre e, em caso de neste período haver mudança de tipo de matéria-prima, o produtor deverá analisar número de amostras correspondente ao número de tipos de matérias-primas utilizadas.

7. A mistura óleo diesel/biodiesel utilizada deverá obedecer aos limites estabelecidos para ponto de entupimento de filtro a frio constantes da especificação vigente da ANP de óleo diesel automotivo.

8. Os métodos referenciados demandam validação para as oleaginosas nacionais e rota de produção etílica.

9. Não aplicáveis para as análises mono-, di-, triglicerídeos, glicerina livre e glicerina total de palmiste e coco. No caso de biodiesel oriundo de mamona deverão ser utilizados, enquanto não padronizada norma da Associação Brasileira de Normas Técnicas - ABNT para esta determinação, os métodos: do Centro de Pesquisas da Petrobrás CENPES constantes do ANEXO B para glicerina livre e total, mono e diglicerídeos, triglicerídeos.

As normas de padronização estabelecemmetodologias para a determinação de alguns dos parâmetros de qualidade do biodiesel. Entretanto, vários métodos alternativos foram propostos e estão descritos na literatura.

Com o uso de biodiesel na matriz energética mundial é necessário considerar os vários aspectos envolvidos na influência desse biocombustível sobre as propriedades dos diversos materiais que compõem peças de motores e os sistemas de transporte e armazenamento.

Nesse artigo serão abordadas as principais técnicas analíticas propostas para o monitoramento da reação de transesterificação, para a determinação do conteúdo dos ésteres graxos e das principais impurezas que possam estar presentes no biodiesel, assim como alguns métodos usados para a determinação do conteúdo de biodiesel em suas misturas com diesel fóssil. Também serão descritos os estudos iniciais de avaliação do efeito do biodiesel sobre materiais poliméricos e metálicos que compõem peças de motores, tanques de armazenagem e transporte.

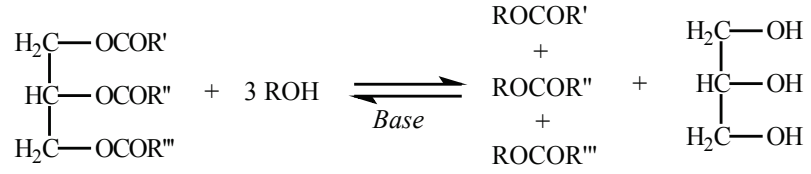

Figura 1: Reação de transesterificação de triglicerídeos oriundos de óleos e gorduras 


\section{MONITORAMENTO DA REAÇÃO DE TRANSESTERIFICAÇÃO}

Baixataxadeconversãonareaçãode transesterificação, reações paralelas e purificação inadequada durante o processo produtivo de biodiesel são alguns dos fatores que influenciam a quantidade de ésteres graxos, glicerol, mono-, di-, triglicerídeos, álcool e catalisador residual no produto final. Especialmente, os conteúdos de mono- e diglicerídeos só podem ser controlados durante a reação de transesterificação, evidenciando a importância do monitoramento desta etapa. ${ }^{13}$

Vários tipos de métodos cromatográficos foram propostos para o acompanhamento da reação de transesterificação. A cromatografia em camada delgada (CCD) analítica foi um dos primeiros métodos empregados para essa análise. ${ }^{15,16}$ Ela é uma técnica rápida e eficiente que permite a análise dos ésteres graxos, tri-,di- e monoglicerídeos. Entretanto, ela é uma técnica especialmente qualitativa, não sendo muito exata quando utilizada para a quantificação dos componentes, e que não permite, portanto, a determinação exata da conversão de reação.

A técnica mais utilizada para o monitoramento da transesterificação é, sem dúvida, a cromatografia gasosa (CG) com detecção por FID (Flame Ionization Detection). Nos métodos desenvolvidos, ${ }^{17-20}$ empregam-se colunas capilares de sílica e a amostra deve ser derivatizada para que os acil-derivados do glicerol possam ser identificados. A derivatização costuma envolver uma reação de sililação com um reagente como o BSTFA [ $\mathrm{N}, \mathrm{O}$-bis(trimetilsilil) trifluoracetamida]. A técnica de $\mathrm{CG}$ é bastante eficiente para a quantificação tanto dos ésteres graxos, quanto dos mono-, di- e triglicerídeos.

A cromatografia líquida de alta eficiência (CLAE) também pode ser utilizada na identificação dos mesmos compostos na mistura reacional. Diferentes métodos de separação (fase reversa, exclusão, etc) assim como distintos modos de detecção (UV, ELSD - Evaporative Light Scattering Detection, EM - Espectrometria de Massas) foram usados. ${ }^{21,22}$ A principal vantagem da CLAE em relação CG é que não há necessidade de derivatização da amostra antes da sua análise.

Além dos métodos cromatográficos, o uso de métodos espectroscópicos também foi proposto a fim de acompanhar a reação de transesterificação. A ressonância magnética nuclear (RMN) é mais rápida e simples que as técnicas cromatográficas, assim como requer uma menor quantidade de amostra, que não precisa de pré-purificação. Tanto a RMN de ${ }^{1} \mathrm{H}$ quanto a RMN de 13C foram propostas para o acompanhamento da transesterificação com metanol ou etanol. ${ }^{23-26} \mathrm{~A}$ desvantagem do uso da RMN são os custos relativamente altos de instrumentação e de manutenção. A espectroscopia no infravermelho (IR) também é amplamente usada para o monitoramento da reação. Para tal, emprega-se a região do infravermelho próximo (NIR), ${ }^{27,28}$ do infravermelho médio ${ }^{29,30}$ e a espectroscopia Raman. ${ }^{31}$ Além disso, sua correlação com outras técnicas permite a verificação da qualidade do biodiesel, ${ }^{28}$ com a quantificação, por exemplo de metanol residual. Entretanto, a determinação dos compostos minoritários nos níveis estabelecidos nas normas de padronização não é possível por este tipo de espectroscopia. ${ }^{28}$

\section{DETERMINAÇÃO DE ÉSTERES GRAXOS E OUTROS COMPOSTOS EM BIODIESEL}

O conteúdo de ésteres graxos do biodiesel é o principal parâmetro na averiguação da qualidade do biodiesel. As normas internacionais estabelecem que ele deve estar acima de 96,5 \%; valores inferiores, além de afetar os demais parâmetros regulamentados, podem provocar o entupimento de filtros de combustível e prejuízos ao motor, devido à polimerização dos triglicerídeos não reagidos. ${ }^{13}$ Fernando et al. ${ }^{14}$ ressaltam a importância da averiguação da influência do baixo conteúdo de ésteres sobre os demais parâmetros de qualidade, assim como seu efeito sobre a durabilidade e a performance dos motores. Em geral, mesmo com um baixo conteúdo de ésteres, várias especificações são atendidas, o que evidencia a importância da análise do teor de ésteres.

O biodiesel é composto majoritariamente por ésteres mono-alquílicos graxos, mas outras impurezas, tais como água, álcool, ácidos graxos, catalisador residual, glicerol e seus acil-derivados, também podem estar presentes. Altas concentrações de glicerol, mono-, di- e trilgicerídeos podem danificar o motor ou mesmo liberar substâncias tóxicas quando da queima do combustível. Eles podem contaminar o biodiesel devido à transesterificação incompleta ou purificação insuficiente do biodiesel. Segundo Knothe ${ }^{28}$ e Mittelbach, ${ }^{12}$ a quantidade desses 
contaminantes é um dos fatores principais para a determinação da qualidade do biodiesel.

O conteúdo de água no biodiesel também é um aspecto importante desde que ela pode promover o crescimento microbiano, causar corrosão em tanques e motores, formar emulsão, causar hidrólise e oxidação hidrolítica do biodiesel. Os métodos baseados em normas de padronização estabelecem o uso de centrifugação e da titulação de Karl-Fischer para sua determinação. ${ }^{11}$

A quantidade de álcool residual no biodiesel também é limitada nas normas de padronização e está intimamente relacionada com o ponto de fulgor. ${ }^{11}$ Quanto maior a quantidade de álcool presente, menor o ponto de fulgor do biodiesel. A presença de álcool também pode ocasionar corrosão metálica dos componentes de motores e tanques de estocagem e transporte assim como acelerar a deteriorização de selos e vedações constituídos de borrachas naturais. ${ }^{13}$

A acidez do biodiesel está relacionada principalmente ao seu conteúdo de ácidos graxos livres. Tal parâmetro também é definido pela ANP e pelas normas internacionais.

O monitoramento da quantidade de metais, tais como Na e K, no biodiesel é necessário devido a questões ambientais e de prejuízos ao motor. As normas de padronização definem a espectrometria de absorção atômica para determinação de tais espécies em biodiesel.

Assim como para o monitoramento da transesterificação, a determinação dos ésteres graxos e dos acil-derivados do glicerol presentes no biodiesel é principalmente realizada por CG. De fato, as técnicas estabelecidas nas normas de padronização brasileira e internacionais estão baseadas no uso de CG-FID para a quantificação de ésteres graxos e do conteúdo de glicerina "livre" e total. Segundo Mittelbach, ${ }^{12}$ somente esta técnica possui todos os requisitos para a determinação do baixo conteúdo destes compostos em biodiesel. Além disso, vários trabalhos na literatura ${ }^{32-38}$ descrevem o uso de $\mathrm{CG}$, com diversos modos de detecção, para o mesmo fim. Em tais análises, emprega-se geralmente coluna capilar e a amostra deve ser previamente derivatizada, assim como padrões têm que ser usados para a quantificação.

O uso de CLAE para a quantificação de ésteres graxos, glicerol e seus derivados em biodiesel também foi proposta em diversos trabalhos. ${ }^{39-45}$ Vários métodos de detecção (ELSD, EM, etc) foram empregados com sucesso.

Uma comparação entre as técnicas CG-EM e CLAEELSD para determinação dos glicerídeos em biodiesel mostrou que ambas são similares, mas CLAE, sob o ponto de vista operacional, é mais adequada por ser aplicável a quase todo tipo de biodiesel, não precisar de derivatização prévia da amostra e ter um tempo de análise menor. ${ }^{46} \mathrm{As}$ técnicas CG e CLAE também podem ser utilizadas em conjunto para análise do glicerol e seus derivados. Essa combinação reduz a complexidade dos cromatogramas de CG, permitindo uma maior confiabilidade na identificação dos picos. Lechner et al. ${ }^{47}$ por exemplo, usaram CLAEDAD (Diode Array Detection) em conjunto com CG-FID para a determinação de mono-, di- e triglicerídeos em amostras de biodiesel metílico.

Um método baseado em eletroforese capilar (EC) com DAD também foi desenvolvido para quantificação de glicerol em biodiesel. ${ }^{48}$ Esse tipo de técnica cromatográfica é bastante rápida, simples e confiável.

A espectroscopia também é muito adequada para a determinação do conteúdo de glicerol em biodiesel. Um método espectrofotométrico baseado na medida da 3,5-diacetil-1,4-diidrolutidina a $410 \mathrm{~nm}$ foi desenvolvido para a determinação do glicerol. ${ }^{49}$ Esse composto é obtido através da reação de Hantzsch do glicerol. Esse método é simples, rápido, econômico e bastante confiável. Esse tipo de análise pode ser utilizado na determinação de biodiesel em diesel, apesar de que a robustez e a aplicabilidade do método a essa matriz ainda devem ser avaliadas.

Além dos métodos cromatográficos e espectroscópicos, técnicas baseadas em propriedades físico-químicas também foram desenvolvidas para a determinação do conteúdo de ésteres em biodiesel. O trabalho de Filippis et al. ${ }^{50}$ descreve o uso de medidas de viscosidade para avaliação do conteúdo de ésteres metílicos em biodiesel. Essas medidas são possíveis já que há uma relação inversa entre a concentração dos ésteres e a viscosidade do meio. Segundo os autores, o método é rápido, simples e especialmente útil para o controle do processo produtivo.

Para a determinação de glicerol "livre" e total em biodiesel são descritos ainda o uso de titulação com 
periodato $^{44}$ e um método enzimático. ${ }^{51} \mathrm{O}$ primeiro apresentou alta precisão e exatidão e o segundo foi muito complexo e com baixa reprodutibilidade. Recentemente, a Sigma-Aldrich Fine Chemicals desenvolveu um kit (BQP-02) para determinação de glicerol total e "livre" em biodiesel, que está baseado em uma reação enzimática e em medidas espectrométricas.

\section{DETERMINAÇÃO DO CONTEÚDO DE BIODIESEL EM MISTURA COM DIESEL}

A determinação do conteúdo de biodiesel em misturas com diesel é geralmente realizada através de métodos espectroscópicos, já que técnicas cromatográficas não são adequadas devido a complexidade da composição química do diesel. ${ }^{52} \mathrm{~A}$ legislação brasileira e as normas internacionais estabelecem o uso da espectroscopia no infravermelho médio (FT-IR) para tal quantificação.

$\mathrm{Na}$ literatura, vários trabalhos também descrevem o uso da espectroscopia no infravermelho médio e próximo. ${ }^{52-56}$ As vantagens do uso desta técnica são a facilidade e a rapidez de análise assim como a manutenção da integridade da amostra. Além disso, os resultados obtidos podem ser correlacionados com RMN para confirmação dos resultados. ${ }^{52}$

Embora o uso de métodos espectroscópicos seja bastante comum, alguns trabalhos descrevem o emprego de técnicas cromatográficas para a determinação das misturas biodiesel/diesel. Em geral, quando a mistura é analisada por CG-FID, realiza-se um pré-tratamento da amostra, que pode envolver uma reação de derivatização e um processo de extração. Apesar da necessidade do prétratamento, os métodos de CG descritos ${ }^{57,58}$ apresentaram boa precisão e exatidão. Além disso, Bondioli et al. ${ }^{57}$ puderam também correlacionar os dados obtidos por CG com o índice de saponificação, permitindo, portanto a determinação da concentração do biodiesel a partir dessa simples medida.

Métodos de CLAE em fase normal, com detectores UV, DAD, ELSD e RID (Refractive Index Detector) foram também descritos para quantificação de biodiesel em diesel. ${ }^{59,60}$ Preferencialmente o detector ELSD deve ser usado, já que sua resposta está diretamente relacionada com a massa do soluto injetada. ${ }^{59}$ Segundo os autores, ${ }^{59} \mathrm{o}$ método é rápido e pode ser usado para a quantificação de qualquer biodiesel em diesel.

\section{EFEITO DO BIODIESEL SOBRE MATERIAIS METÁLICOS E POLIMÉRICOS}

Como o biodiesel é considerado um combustível alternativo proeminente, a resistência de algumas partes dos motores deve ser avaliada para que materiais compatíveis possam ser utilizados. ${ }^{13}$

Ainda existem poucos estudos de avaliação do efeito do biodiesel sobre os materiais utilizados em motores ciclo diesel e nos sistemas de transporte e armazenamento. $\mathrm{O}$ biodiesel solubiliza facilmente certos elastômeros e materiais depositados nos tanques, o que pode ocasionar entupimento de filtros de combustíveis e do sistema de injeção. ${ }^{61} \mathrm{O}$ uso de B100, mesmo dentro das especificações, pode acelerar o desgaste de selos, mangueiras e vedações dos motores. Materiais tais como borracha natural e nitrílica, propileno, polivinil e Tygon são bastante vulneráveis e o seu uso deve ser evitado. ${ }^{13}$ Poliamidas, poli(oxido de metileno), poli(tereftalato de butileno) e poli(sulfeto de fenileno) não foram afetados quando em contato com biodiesel de soja, colza e palma, sendo que o PPS foi o mais resistente. ${ }^{62}$

Misturas de biodiesel com diesel também atacam certos materiais. No trabalho de Trakarnpruk e Porntangjitlikit, ${ }^{63}$ foram avaliados 6 tipos de elastômeros (NBR, HNBR, NBR/PVC, borracha acrílica, co-polímero FKM e terpolímero FKM) em testes de imersão em B10. Os materiais NBR, NBR/PVC e a borracha acrílica foram mais suscetíveis ao ataque que os demais. Os elastômeros fluoretados (FKM) foram os menos afetados. Portanto, os estudos realizados até o momento indicam que elastômeros fluoretados assim como aço inoxidável e alumínio são materiais compatíveis com o biodiesel ${ }^{61,64}$ e o uso de Teflon, Viton e Nylon é recomendado. ${ }^{13}$ Os estudos também indicam que o alto conteúdo de compostos insaturados no biodiesel aumenta o seu potencial corrosivo. Além disso, observou-se também que os materiais metálicos afetam a constituição química do biodiesel, o que pode ser verificado pelo aumento no seu índice de acidez. ${ }^{61}$ Bronze, latão, cobre, chumbo, estanho e zinco, ferro e níquel são incompatíveis com o biodiesel, diminuindo sua estabilidade oxidativa $^{61,65} \mathrm{e}$, por conseqüência, aumentando sua ação corrosiva. $\mathrm{O}$ efeito corrosivo do biodiesel também é maior quando a quantidade de água e metanol presentes no biodiesel está acima dos limites especificados nas normas de padronização. ${ }^{11,66}$ 


\section{CONSIDERAÇÕES FINAIS}

A aplicação de métodos analíticos na produção, armazenamento, distribuição e produto final (em misturas com diesel fóssil) é de fundamental importância para a avaliação e controle da qualidade do produto. Este controle, estabelecido por meio de especificações técnicas normatizadas, define os limites de aceitação de cada parâmetro físico-químico avaliado. Além disso, os diversos métodos de análise para biodiesel permitem o monitoramento da reação de transesterificação e dos produtos formados no decorrer do processo, bem como a determinação do conteúdo de biodiesel em misturas com diesel fóssil; fatores de suma importância na avaliação da qualidade do produto.

Métodos cromatográficos e espectroscópicos são as técnicas mais utilizadas para a caracterização do biodiesel, mas também são descritos procedimentos baseados em propriedades físicas. Especificamente, a cromatografia gasosa (CG) e a cromatografia líquida de alta eficiência (CLAE) são utilizadas para a determinação do conteúdo de ésteres monoalquílicos graxos, ácidos graxos, glicerol, mono-, di- e triglicerídeos em biodiesel, porém CG-FID é a técnica mais utilizada em virtude da sua exatidão na determinação de componentes minoritários. Entretanto, o uso da detecção por EM é útil para a identificação inequívoca do analito. A caracterização do biodiesel por CLAE é menos utilizada, mas possui algumas vantagens, tais como menor tempo de análise e a não necessidade de derivatização prévia da amostra.

Para o monitoramento da reação de transesterificação, utiliza-se com freqüência CG-FID, mas os métodos espectroscópicos, tais como RMN e IR são também reportados para esta finalidade. Por outro lado, a determinação do conteúdo de biodiesel nas misturas biodiesel/diesel é realizada principalmente por métodos espectroscópicos (RMN e IR) devido à grande variabilidade de compostos químicos presentes no diesel, que tornam os cromatogramas bastante complexos.

A comercialização de biodiesel implica no seu uso em motores e em sistemas de transporte e armazenamento atualmente utilizados para o diesel fóssil. Desta forma, o efeito desse biocombustível sobre os materiais que constituem esses sistemas é um fator de suma importância. Entretanto, poucos estudos são reportados sobre o impacto do biodiesel (e suas misturas com óleo diesel) sobre os materiais atualmente empregados pela indústria automobilística e de autopeças. Os resultados obtidos até o momento indicam que o uso de alguns elastômeros e metais deve ser evitado. Além disso, a presença de ésteres com muitas insaturações, assim como de água e metanol no biodiesel potencializam seu efeito corrosivo sobre materiais metálicos. Entretanto, a maioria dos resultados obtidos refere-se a biodiesel originário da colza (variedade da canola), evidenciando a necessidade de estudos com biodiesel oriundo de oleaginosas brasileiras, para que se possa estabelecer, por exemplo, a relação entre a composição química do biodiesel e o seu efeito sobre os materiais utilizados pelos fabricantes de motores e auto-peças. O desenvolvimento de materiais compatíveis à matriz energética, onde está inserido o biodiesel, tem se configurado como um campo de pesquisa bastante promissor, bem como a busca por métodos analíticos mais simples e de menor custo para o controle da qualidade do biodiesel.

\section{REFERÊNCIAS}

1. Agarwal, A. K.; Prog. Energ. Combust. 2007, 33, 233.

2. International Energy Agency (IEA), Biofuels for transport: an international perspective, IEA/EET, Paris, maio, 2004.

3. Tan, M. E.; Van Gerpen, J. H.; J. Am. Oil Chem. Soc. 1999, 76, 1511.

4. Encinar, J. M.; González, J. F.; Reinares, A. R.; Fuel Process. Technol. 2007, 88, 513.

5. Dantas, M. B.; Almeida, A. A. F.; Conceição, M. M.; Fernandes Jr., V. J.; Santos, I. M. G.; Silva, F. C.; Soledade, L. E. B.; Souza, A. G.; J. Therm. Anal. Cal. 2007, 87, 847.

6. Biodiesel: barreiras, potenciais e impactos, http:// www.esru.strath.ac.uk/EandE/Web_sites/06-07/ Biodiesel/biodieselp.htm, acesso em 27/08/2007.

7. Knothe, G.; Trans. ASAE 2001, 44, 193.

8. Ma, F.; Hanna, M. A.; Bioresource Technol. 1999, 70,1 . 


\section{Artigo 3}

9. Pinto, A. C.; Guarieiro, L. L. N.; Rezende, M. J. C.; Ribeiro, N. M.; Torres, E. A.; Lopes, W. A.; Pereira, P. A. P.; de Andrade, J. B.; J. Braz. Chem. Soc. 2005, 16, 1313.

10. Meher, L. C.; Sagar, D. V.; Naik, S. N.; Renew. Sustain. Energy Rev. 2006, 10, 248.

11. Knothe, G.; J. Am. Oil. Chem. Soc. 2006, 83, 823.

12. Mittelbach, M.; Bioresour. Technol. 1996, 56, 7.

13. Abdullah, A. Z.; Razali, N.; Mootabadi, H.; Salamatinia, B.; Environ. Res. Lett. 2007, $2,1$.

14. Fernando, S.; Karra, P.; Hernandez, R.; Jha, S. K.; Energy 2007, 32, 844.

15. Freedman, B.; Pryde, E. H.; Kwolek, W. F.; J. Am. Oil Chem. Soc. 1984, 61, 1215.

16. Cvengros, J.; Cvengrosova, Z.; Hoka, C.; Petrol. Coal 2002, 44, 67.

17. Freedman, B.; Kwolek, W. F.; Pryde, E. H.; J. Am. Oil Chem. Soc. 1986, 63, 1370.

18. Cvengros, J.; Cvengrosova, Z.; J. Am. Oil Chem. Soc. 1994, 71, 1349.

19. Cvengrosova, Z.; Cvengros, J.; Hronec, M.; Petrol. Coal 1997, 39, 36.

20. Cvengrosova, Z.; Cvengros, J.; Hronec, M.; Petrol. Coal 1998, 40, 97.

21. Trathnigg B.; Mittelbach, M.; J. Liq. Chromatogr. 1990, 13, 95.

22. Holcapek, M.; Jandera, P.; Fischer, J.; Prokes, B.; J. Chromatogr. A 1999, 858, 13.

23. Gelbard, G.; Bres, O.; Vargas, R. M.; Vielfaure, F.; Schuchardt, U.F.; J.Am. Oil Chem. Soc. 1995, 72, 1239.

24. Dimmig, T.; Radig, W.; Knoll, C.; Dittmar, T.;

Chemische Technik (Chem. Tech.) 1999, 51, 326.

25. Costa Neto, P. R.; Caro, M. S. B.; Mazzuco, L. M.; Nascimento,M.G.;J.Am.OilChem.Soc.2004,81,1111.

26. Morgenstern, M.; Cline, J.; Meyer, S.; Cataldo, S.; Energy Fuels 2006, 20, 1350.
27. Knothe, G.; J. Am. Oil Chem. Soc. 1999, 76, 795.

28. Knothe, G.; J. Am. Oil Chem. Soc. 2000, 77, 489.

29. Zagonel, G. F.; Peralta-Zamora, P.; Ramos, L. P.; Talanta 2004, 63, 1021.

30. Siatis, N. G.; Kimbaris, A. C.; Pappas, C. S.; Tarantilis, P. A.; Polissiou, M. G.; J. Am. Oil Chem. Soc. 2006, 83, 53.

31. Ghesti, G. F.; de Macedo, J. L.; Braga, V. S.; de Souza, A. T. C. P.; Parente, V. C. I.; Figueredo, E. S.; Resck, I. S.; Dias, J. A.; Dias, S. C. L.; J. Am. Oil Chem. Soc. 2006, 83, 597.

32. Mariani, C.; Bondioli, P.; Venturini, S.; Fedeli, E.; Riv. Ital. Sostanze Grasse 1991, 68, 549.

33. Wawrzyniak, R.; Wasiak, W.; Frackowiak, M.; Chem. Pap. 2005, 59, 449.

34. Schober, S.; Seidl, I.; Mittelbach, M.; Eur. J. Lipid Sci. Technol. 2006, 108, 309.

35. Bondioli, P.; Mariani, C.; Lanzani, A.; Fedeli, E.; Veronese, S.; Riv. Ital. Sostanze Grasse 1992, 69, 7.

36. Plank C.; Lorbeer, E.; J. High Res. Chromatog. 1992, 15, 609 .

37. Mittelbach, M.; Chromatographia 1993, 37, 623.

38. Mittelbach, M.; Roth, G.; Bergmann, A.; Chromatographia 1996, I, 431.

39. Fillieres, R.; Mlayah, B. B.; Delmas, M.; J. Am. Oil Chem. Soc. 1995, 72, 427.

40. Darnoko, D.; Cheryan, M.; Perkins, E. G.; J. Liq. Chromatogr. Relat. Technol. 2000, 23, 2327.

41. Foglia, T. A.; Jones, K. C.; J. Liq. Chromatogr. Relat. Technol. 1997, 20, 1829.

42. Holcapek, M.; Jandera, P.; Adv. Mass Spectrom. 2001, 15, 583.

43. Lozano, P.; Chirat, N.; Graille, J.; Pioch, D.; Fresenius'J. Anal. Chem. 1996, 354, 319.

44. Sala, M.; Bondioli, P.; Riv. Ital. Sostanze Grasse 1998, 75, 305. 
45. Hajek, M.; Skopal, F.; Machek, J.; Eur. J. Lipid Sci. Technol. 2006, 108, 666.

46. Foglia, T. A.; Jones, K. C.; Nunez, A.; Phillips, J. G.; Mittelbach, M.; Chromatographia 2004, 60, 305.

47. Lechner, M.; Plank, C.; Lorbeer, E.; J. High Res. Chromatog. 1997, 20, 581.

48. Gonçalves Filho, L. C.; Micke, G. A.; J. Chromatogr. A 2007, 1154, 477.

49. Bondioli, P.; Della Bella, L.; Eur. J. Lipid Sci. Technol. 2005, 107, 153.

50. Filippis, P.; Giavarini, C.; Scarsella, M.; Sorrentino, M.; J. Am. Oil Chem. Soc. 1995, 72, 1399.

51. Bailer, J.; de Hueber, K.; Fresenius' J. Anal. Chem. 1991, 340, 186.

52. Knothe, G.; J. Am. Oil Chem. Soc. 2001, 78, 1025.

53. Birova, A.; Svajdlenka, E.; Cvengros, J.; Dostalikova, V.; Eur. J. Lipid Sci Technol. 2002, 104, 271.

54. Oliveira, J. S.; Montalvão, R.; Daher, L.; Suarez, P. A. Z.; Rubim, J. C.; Talanta 2006, 69, 1278.

55. Pimentel, M. F.; Ribeiro, G. M. G. S.; da Cruz, R. S.; Stragevitch, L.; Pacheco Filho, J. G. A.; Teixeira, L. S. G.; Microchem. J. 2006, 82, 201.

56. Aliske, M. A.; Zagonel, G. F.; Costa, B. J.; Veiga, W.; Saul, C. K.; Fuel 2007, 86, 1461.

57. Bondioli, P.; Lanzani, A.; Fedeli, E.; Sala, M.; Veronese, S.; Riv. Ital. Sostanze Grasse 1994, 71, 287.

58. Bondioli, P.; Della Bella, L.; Riv. Ital. Sostanze Grasse 2003, 80, 173.
59. Foglia, T. A.; Jones, K. C.; Phillips, J. G.; Chromatographia 2005, 62, 115.

60. Kaminski, M.; Gilgenast, E.; Przyjazny, A.; Romanik, G.; J. Chromatogr. A 2006, 1122, 153.

61. Kaul, S.; Saxena, R. C.; Kumar, A.; Negi, M. S.; Bhatnagar, A. K.; Goyal, H. B.; Gupta, A. K.; Fuel Process. Technol. 2007, 88, 303.

62. Herrero, R.; Maiza, I.; Landa, I.; Revista de Plasticos Modernos 2006, 92, 147.

63. Trakarnpruk, W.; Porntangjitlikit, S.; Renew. Energy 2007, in press.

64. Graboski, M. T.; McCornick, R. L.; Prog. Energy Combust. Sci. 1998, 24, 125.

65. Knothe, K.; Dunn, R. O.; J. Am. Oil Chem. Soc. 2003, 80, 1021.

66. Cvengros, J.; Fett/Lipid 1998, 100, 41.

\section{Luciano M. Lião*1, Marcos R. Monteiro ${ }^{2}$ e Alessandra R. P. Ambrozin ${ }^{2}$}

${ }^{1}$ Instituto de Química, UFG, Campus Samambaia, CP 131, CEP 74001-970, Goiânia, GO, Brasil

${ }^{2}$ Laboratório de Combustíveis, Centro de Caracterização e Desenvolvimento de Materiais, Departamento de Engenharia de Materiais, UFSCar, CEP 13560-971, São Carlos, SP, Brasil

"E-mail: luciano@quimica.ufg.br 